\title{
SARS-CoV-2 and the rheumatology patient: the last 12 months and a boost in the future
}

\author{
Kevin L Winthrop (ㄷ), ${ }^{1}$ Richard J Whitley, ${ }^{2}$ Daniel Aletaha (i] ${ }^{3}$
}

\section{EDITORIAL}

It is a rare opportunity to enter the backside (hopefully) of a pandemic. Not since the Spanish influenza has the world experienced such a level of contagion. While we averted a worldwide crisis 20 years ago with the first SARS virus infection, SARS-CoV-2 with its unique ability to transmit easily among asymptomatic persons has altered our 21st-century appreciation and respect for viral diseases. From a scientific standpoint, we believe the scientific collaboration and innovation of the last 12 months have been unprecedented. The pandemic united rheumatologists and infectious disease physicians in an effort to develop both therapeutics and vaccines. While some of our patients appear to be partially protected with the currently available vaccines, we must continue our efforts at understanding optimal ways to manage Disease Modifying Anti-Rheumatic Drug (DMARD) therapy and vaccination in light of COVID-19. Providing a booster dose of vaccine to those with suboptimal vaccine responses, particularly those at greatest risk due to immune compromise, must urgently be pursued and evaluated.

In this themed issue of the Annals of the Rheumatic Diseases, focused on COVID-19 and SARS-CoV-2 vaccination in rheumatology, a number of papers have been assembled, which advance our understanding in this area and should allow for optimising the approach to this pandemic in the near future. ${ }^{1-20}$ To date, our understanding of COVID-19 risk in rheumatology comes primarily from observational registry-based cohorts. A clear signal has emerged in that patients receiving B-cell depletion therapy or

\footnotetext{
'Division of Infectious Diseases, and School of Public Health, Oregon Health \& Science University, Portland, Oregon, USA

${ }^{2}$ Division of Infectious Diseases, The University of Alabama at Birmingham School of Medicine, Birmingham, Alabama, USA

${ }^{3}$ Department of Rheumatology, Medical University of Vienna, Vienna, Austria
}

Correspondence to Dr Kevin L Winthrop, Oregon Health \& Science University, Portland, OR 97239, USA; winthrop@ohsu.edu high-dose glucocorticoids at baseline are at higher risk of more severe COVID-19 outcomes if infected. ${ }^{14}$ In addition, and most recently, the global alliance data suggest an increased risk for Janus kinase (JAK) inhibitors as well, although less statistically sophisticated analysis from inflammatory bowel disease registries does not suggest an increased risk for tofacitinib. ${ }^{21}$ Similarly, the findings of these studies are affected by channelling bias/ confounding by indication (eg, those with higher disease activity are more likely to be using these agents), and it is clear that many rheumatology patients who perceive themselves at higher risk are more likely to practise avoidance behaviour to minimise the risk of infection. ${ }^{22}$ Despite the difficulty in controlling for these factors within these studies, there is strong biologic plausibility as to why these drug classes could diminish antiviral host defences. The development of a neutralising antibody response is clearly important in recovery from an initial infection and protection from subsequent infection. ${ }^{23-25}$ Furthermore, interferon signalling is an essential host response to a number of viral infections, including SARS-CoV-2. ${ }^{26}$

\section{THERAPEUTICS}

The rheumatological therapeutic armamentarium took centre stage from the beginning of the pandemic, with an effort to repurpose existing drugs for both antiviral and anti-inflammatory purposes. Despite the 'Trumped-up' early results of hydroxychloroquine studies, randomised controlled trials (RCTs) provided no evidence of efficacy. Despite initial observational studies suggesting efficacy, ${ }^{27}$ the eventual triumph of interleukin 6 inhibition after multiple negative RCTs was notable, ${ }^{28}$ whereby a small magnitude of effect could only be 'significant' with an RCT of unusually large proportions. The Recovery trial enrolled $>4000$ hospitalised hypoxic patients with COVID-19 randomised to tocilizumab or standard of care and observed a decrease in mortality from $33 \%$ to $29 \%$ (corresponding to a number needed to treat of 25$)^{29}$; this was consistent across other similar RCTs when subjected to meta-analysis (OR for survival, 0.83 (0.74-0.92)), ${ }^{30}$ but taken as individual 'pivotal' clinical trials, these studies were deemed 'negative', lacking the statistical power to detect a relatively small magnitude of effect. The evolution of study end points and inclusion criteria across these studies is beyond the scope of this editorial, but suffice it to say, trials eventually settled on the prevention of severe disease (ie, a combined outcome of mechanical ventilation and death) as a primary outcome measure. To date, monoclonal antibodies provide our best antiviral approach (more below), and more recently, from a drug class of initial concern (JAK inhibition) in potentially diminishing host antiviral response, baricitinib has been shown to shorten time to clinical recovery when used with remdesivir and to reduce mortality when used in combination with dexamethasone. ${ }^{31} 32$ In addition, tofacitinib was more recently shown to do much the same. ${ }^{33}$ Thank you Rheumatologists for leading the way!

\section{VACCINATION}

While we lack well-defined 'immune correlates of protection', experimental studies in non-human primates suggest the importance of both cell-mediated and humoral vaccine responses. Use of monoclonal antibodies in infected naïve macaques was protective in dosedependent fashion following infectious challenge. Antibodies limited both the risk of infection and the extent/length of disease among those infected. Regarding T-cell immunity, when comparing previously infected macaques, those that were experimentally depleted of CD8 $+\mathrm{T}$ lymphocytes $(n=5)$ were easily reinfected on challenge compared with macaques with intact cell-mediated immunity $(n=5)$ that did not develop infection. ${ }^{34}$ These data lay the foundation for our observations in human RCTs of monoclonal antibody therapy against SARS-CoV-2-data from pivotal phase III vaccination studies, as well as observational studies of breakthrough infections among those previously vaccinated; taken together, these data inform strategies as to how to best to manage the rheumatology patient in this pandemic era.

We now have human data that recapitulate those from the experimental macaque studies. Prophylactic use of anti-SARS-CoV-2 monoclonal antibodies among uninfected humans shows the ability to prevent infection. ${ }^{35}$ Furthermore, among those infected who have 
not yet mounted an antibody response, neutralising antibodies have proven able to prevent progression of infection from mild to severe disease. ${ }^{3536}$ The development of these antibodies started with high-dose products, but eventually proved that lower doses were equivalent in antiviral capacity. These data suggest that some threshold level is necessary to provide protection; however, this threshold remains undefined and might differ depending on whether cell-mediated immune responses are present. The data from phase III vaccine studies attest to a correlation of high vaccine efficacy with both robust humoral and T-cell responses. ${ }^{37}$ Importantly, diminished efficacy against some variants correlates with in vitro reductions in vaccine-induced neutralising capacity against these variants. ${ }^{38} 39$ Finally, while little published data exist on 'breakthrough' infections to date, early reports suggest immunocompromised patients are disproportionately affected. Up to $40 \%$ of a large cohort $(n=152)$ of breakthrough infections in recipients of the Pfizer messenger RNA (mRNA) vaccine in Israel were immunocompromised, and B-cell depletion therapy was an important identified risk factor. ${ }^{40}$

To date, studies from rheumatic diseases, transplant, glomerular diseases and multiple sclerosis have consistently identified a large percentage of vaccinated individuals who have no measurable humoral responses after vaccination. ${ }^{41}$ B-cell depletion therapy, mycophenolate, tacrolimus and high-dose corticosteroids have all been associated with a lack of seroconversion. ${ }^{62}{ }^{43}$ Interestingly, some data suggest that patients using rituximab still develop cell-mediated immunity despite a lack of humoral response. ${ }^{44}$ Methotrexate has been shown to strongly diminish the development of cytotoxic CD8+ responses, documented to be important in SARS-CoV-2 protection at least among macaques. ${ }^{8} 34$ There are data suggesting JAK inhibitors and tumour necrosis factor blockers also diminish responses, but to much lesser degrees. ${ }^{42}$

\section{BOOST OR NOT TO BOOST}

As we write, the American College of Immunisation Practices is meeting to consider this very issue. The demonstrable efficacy of exogenous monoclonal antibodies clearly speaks to the importance of protective neutralising antibody responses so that, in our mind, patients with an undetectable antibody response after vaccination will not likely have the same protection as those with positive titres.
While the level of sufficient or 'protective' titre is unknown, it is hard to believe that an absence of titre does not equate to diminished protection, even if vaccineinduced cell-mediated immune responses are developed. Accordingly, for those individuals receiving B-cell depletion and other therapies strongly associated with a lack of seroconversion, it seems reasonable to evaluate postvaccination titres, recognising such serological assessments are not necessary for otherwise healthy individuals. It is also important to recognise that there is wide variability in the reliability of existing licensed assessments. If antibodies are absent, however, then a booster dose of vaccine will increase the likelihood of their development and ultimately might increase protection. ${ }^{45}$ For all other DMARD recipients, while levels of postvaccination titres might be somewhat diminished, it is unclear whether this is problematic and whether a booster of vaccine would be required or even helpful. In fact, the first study in immunosuppressed non-responders to mRNA vaccination has been completed, in which a randomised comparison of inducible humoral and cellular immune answers to a third booster vaccination with mRNA vaccine versus single switch boost using a vector vaccine was done, and results are awaited eagerly (clinical trial registration number: 2021-002348-57). In the meantime, our patients who are likely to develop inadequate vaccine responses should live like it was 2020, with masking and avoidance in mind, and sceptics need to be reminded the greatest risk of inadequate immune response occurs with a failure to get vaccinated! We should continue to pursue studies to evaluate the effect of holding certain DMARDs to determine whether this assists with the building or maintenance of vaccine-induced immune responses associated with both primary and booster immunisations alike. Finally, we should reassure our patients that the vaccines in use are safe, not associated with underlying disease flare to date and much more enjoyable to receive than COVID-19 itself.7 11141546

\section{Handling editor Johannes WJ Bij|sma}

Contributors KLW, RJW and DA contributed to the creation of this editorial in all aspects including writing and critical revision of the manuscript.

Funding The authors have not declared a specific grant for this research from any funding agency in the public, commercial or not-for-profit sectors.

Disclaimer The opinions endorsed in this editorial are personal opinions of the authors, and do not necessarily reflect official guidance of the various professional organisations in which the authors may hold advisory roles.
Competing interests KLW has received research grants from Pfizer and consultant honorarium from Lilly, Regeneron, Gilead, Pfizer and Astra Zeneca. RW receives grant support from the NIH and serves as the Chair of NIAID's DSMBs for Coronavirus Vaccine Studies and for HIV. He is on the Board of Directors of Viros. DA received research grants from Abbvie, Amgen, Lilly, Novartis, Roche, SoBi and Sanofi, and consultant/ speaker honorarium from Abbvie, Amgen, Lilly, Merck, Novartis, Pfizer, Roche and Sandoz.

Patient and public involvement Patients and/or the public were not involved in the design, or conduct, or reporting, or dissemination plans of this research.

Patient consent for publication Not required.

Provenance and peer review Commissioned; externally peer reviewed.

(C) Author(s) (or their employer(s)) 2021. No commercial re-use. See rights and permissions. Published by BMJ.

$$
\text { (D) Check for updates }
$$

To cite Winthrop KL, Whitley RJ, Aletaha D. Ann Rheum Dis 2021;80:1249-1251.

Received 30 July 2021

Accepted 25 August 2021

Ann Rheum Dis 2021;80:1249-1251.

doi:10.1136/annrheumdis-2021-221251

\section{ORCID iDs}

Kevin L Winthrop http://orcid.org/0000-0002-38926947

Daniel Aletaha http://orcid.org/0000-0003-2108-0030

\section{REFERENCES}

1 Bonelli MM, Mrak D, Perkmann T. SARS-CoV-2 vaccination in rituximab-treated patients: evidence for impaired humoral but inducible cellular immune response. Ann Rheum Dis 2021:80:1355-6.

2 Bower H, Frisell T, Di Giuseppe D, et al. Impact of the COVID-19 pandemic on morbidity and mortality in patients with inflammatory joint diseases and in the general population: a nationwide Swedish cohort study. Ann Rheum Dis 2021;80:1086-93.

3 Braun-Moscovici Y, Kaplan M, Braun M. Disease activity and humoral response in patients with inflammatory rheumatic diseases after two doses of the pfizer mRNA vaccine against SARS-CoV-2. Ann Rheum Dis 2021:80:1316-20.

4 Bugatti S, De Stefano L, Balduzzi S. Methotrexate and glucocorticoids, but not anticytokine therapy, impair the immunogenicity of a single dose of the BNT162b2 mRNA COVID-19 vaccine in patients with chronic inflammatory arthritis. Ann Rheum Dis 2021.

5 D'Onofrio B, De Stefano L, Palermo BL, et al. Challenges in the diagnosis of early rheumatoid arthritis in times of COVID-19. Ann Rheum Dis 2021;80:1242-3.

6 Furer V, Eviatar T, Zisman D. Immunogenicity and safety of the BNT162b2 mRNA COVID-19 vaccine in adult patients with autoimmune inflammatory rheumatic diseases and in the general population: a multicentre study. Ann Rheum Dis 2021;80:1329-37.

7 Geisen UM, Berner DK, Tran F. Immunogenicity and safety of anti-SARS-CoV-2 mRNA vaccines in patients with chronic inflammatory conditions and immunosuppressive therapy in a monocentric cohort. Ann Rheum Dis 2021;80:1305-10.

8 Haberman RH, Herati R, Simon D. Methotrexate hampers immunogenicity to BNT162b2 mRNA COVID-19 vaccine in immune-mediated inflammatory disease. Ann Rheum Dis 2021;80:1338-43.

9 Janssen MTHF, Ramiro S, Landewé RBM. Antibody response to SARS-CoV-2 in patients receiving glucocorticoids with or without tocilizumab for COVID 
19-associated hyperinflammation. Ann Rheum Dis 2021:80:1362-3.

10 Melikhov 0, Kruglova T, Lytkina K, et al. Use of Janus kinase inhibitors in COVID-19: a prospective observational series in 522 individuals. Ann Rheum Dis 2021:80:1245-6.

11 Priori R, Pellegrino G, Colafrancesco S, et al. SARSCoV-2 vaccine hesitancy among patients with rheumatic and musculoskeletal diseases: a message for rheumatologists. Ann Rheum Dis 2021;80:953-4.

12 Rimar D, Slobodin G, Paz A, et al. SARS-COV-2 vaccination after stem cell transplantation for scleroderma. Ann Rheum Dis 2021:80:1354-5.

13 Simon D, Tascilar K, Fagni F, et al. SARS-CoV-2 vaccination responses in untreated, conventionally treated and anticytokine-treated patients with immune-mediated inflammatory diseases. Ann Rheum Dis 2021;80:1311-5.

14 Sparks JA, Wallace ZS, Seet AM, et al. Associations of baseline use of biologic or targeted synthetic DMARDs with COVID-19 severity in rheumatoid arthritis: results from the COVID-19 global rheumatology alliance physician registry. Ann Rheum Dis 2021:80:1137-46

15 Strangfeld A, Schäfer M, Gianfrancesco MA, et al. Factors associated with COVID-19-related death in people with rheumatic diseases: results from the COVID-19 global rheumatology alliance physicianreported registry. Ann Rheum Dis 2021;80:930-42.

16 Trahtemberg U, Rottapel R, Dos Santos CC, et al. Anticardiolipin and other antiphospholipid antibodies in critically ill COVID-19 positive and negative patients. Ann Rheum Dis 2021:80:1236-40.

17 Ursini F, Ruscitti P, D'Angelo S, et al. Broad clinical spectrum of SARS-CoV-2-associated inflammatory joint disease in adults: a report of 35 cases from the COVID-19 \& autoimmune systemic disease italian study group. Ann Rheum Dis 2021. doi:10.1136/ annrheumdis-2021-220606. [Epub ahead of print: 26 May 2021].

18 Vijayan A, Ahmed S, Joseph S, et al. Effects of face masks on oxygen saturation and functional measures in patients with connective tissue disorder-associated interstitial lung disease. Ann Rheum Dis 2021. doi:10.1136/annrheumdis-2021-220230. [Epub ahead of print: 10 May 2021].

19 Westhoff TH, Seibert FS, Anft M. Correspondence on 'SARS-CoV-2 vaccination in rituximab-treated patients: evidence for impaired humoral but inducible cellular immune response'. Ann Rheum Dis 2021;80:e162.

20 Wong AY, MacKenna B, Morton CE, et al. Use of nonsteroidal anti-inflammatory drugs and risk of death from COVID-19: an Opensafely cohort analysis based on two cohorts. Ann Rheum Dis 2021;80:943-51.

21 Agrawal M, Brenner EJ, Zhang X, et al. Characteristics and outcomes of IBD patients with COVID-19 on tofacitinib therapy in the secure-IBD registry. Inflamm Bowel Dis 2021:27:585-9.

22 George MD, Venkatachalam S, Banerjee S, et al. Concerns, healthcare use, and treatment interruptions in patients with common autoimmune rheumatic diseases during the COVID-19 pandemic. J Rheumatol 2021:48:603-7.

23 Choi B, Choudhary MC, Regan J, et al. Persistence and evolution of SARS-CoV-2 in an immunocompromised host. N Eng/ J Med 2020;383:2291-3.

24 Friedman MA, Winthrop KL. Second COVID-19 infection in a patient with granulomatosis with polyangiitis on rituximab. Ann Rheum Dis 2021;80:1102-4.

25 Hu F, Chen F, Ou Z, et al. A compromised specific humoral immune response against the SARS-CoV-2 receptor-binding domain is related to viral persistence and periodic shedding in the gastrointestinal tract. Cell Mol Immunol 2020;17:1119-25.

26 Bastard P, Rosen LB, Zhang Q, et al. Autoantibodies against type I IFNs in patients with life-threatening COVID-19. Science 2020:370:eabd4585.

27 Ramiro S, Mostard RLM, Magro-Checa C, et al. Historically controlled comparison of glucocorticoids with or without tocilizumab versus supportive care only in patients with COVID-19-associated cytokine storm syndrome: results of the chiC study. Ann Rheum Dis 2020;79:1143-51.

28 Winthrop KL, Mariette X. To immunosuppress: whom, when and how? that is the question with COVID-19. Ann Rheum Dis 2020;79:1129-31.

29 RECOVERY CG, RECOVERY Collaborative Group. Tocilizumab in patients admitted to hospital with COVID-19 (recovery): a randomised, controlled, openlabel, platform trial. Lancet 2021;397:1637-45.

30 , Shankar-Hari M, Vale CL, et al, WHO Rapid Evidence Appraisal for COVID-19 Therapies (REACT) Working Group. Association between administration of IL-6 antagonists and mortality among patients hospitalized for COVID-19: a meta-analysis. JAMA 2021;326:499-518.

31 Marconi VC, Ramanan AV, de Bono S. Efficacy and safety of baricitinib in patients with COVID-19 infection: results from the randomised, double-blind, placebo-controlled, parallelgroup cov-barrier phase 3 trial. medRxiv 2021 doi:10.1101/2021.04.30.21255934

32 Kalil AC, Patterson TF, Mehta AK, et al. Baricitinib plus remdesivir for hospitalized adults with Covid-19. N Engl J Med 2021;384:795-807.

33 Guimarães PO, Quirk D, Furtado RH, et al. Tofacitinib in patients hospitalized with Covid-19 pneumonia. $N$ Engl J Med 2021:385:406-15.

34 McMahan K, Yu J, Mercado NB, et al. Correlates of protection against SARS-CoV-2 in rhesus macaques. Nature 2021;590:630-4.
35 IDSA. COVID-19 real-time learning network: therapeutics and interventions, 2021. Monoclonal antibodies. Available: https://www.idsociety.org/ covid-19-real-time-learning-network/therapeutics-andinterventions/monoclonal-antibodies/

36 RECOVERY CG, Horby PW, Mafham M. Casirivimab and imdevimab in patients admitted to hospital with COVID-19 (recovery): a randomised, controlled, open-label, platform trial. medRxiv 2021. doi:10.1101/2021.06.15.21258542

37 IDSA. COVID-19 real-time learning network : vaccines overview, 2021. Available: https://www.idsociety. org/covid-19-real-time-learning-network/vaccines/ vaccines-overview/

38 Jongeneelen M, Kaszas K, Veldman D. Ad26.COV2.S elicited neutralizing activity against delta and other SARS-CoV-2 variants of concern. bioRxiv 2021. doi:10.1101/2021.07.01.450707

39 Liu Y, Liu J, Xia H, et al. BNT162b2-elicited neutralization against new SARS-CoV-2 spike variants. N Engl J Med 2021;385:472-4.

40 Brosh-Nissimov T, Orenbuch-Harroch E, Chowers $M$, et al. BNT162 b2 vaccine breakthrough: clinical characteristics of 152 fully vaccinated hospitalized COVID-19 patients in Israel. Clin Microbiol Infect 2021. doi:10.1016/j.cmi.2021.06.036. [Epub ahead of print: 07 Jul 2021].

41 Friedman MA, Curtis JR, Winthrop KL. Impact of disease modifying anti-rheumatic drugs on vaccine immunogenicity in patients with inflammatory rheumatic and musculoskeletal diseases, 2021.

42 Deepak P, Kim W, Paley MA, et al. Glucocorticoids and $B$ cell depleting agents substantially impair immunogenicity of mRNA vaccines to SARS-CoV-2 medRxiv 2021. doi:10.1101/2021.04.05.21254656. [Epub ahead of print: 09 Apr 2021]. 2021.

43 Itzhaki Ben Zadok O, Shaul AA, Ben-Avraham B, et al. Immunogenicity of the BNT162b2 mRNA vaccine in heart transplant recipients - a prospective cohort study. Eur J Heart Fail 2021. doi:10.1002/ejhf.2199. [Epub ahead of print: 08 May 2021].

44 Mrak D, Tobudic S, Koblischke M, et al. SARS-CoV-2 vaccination in rituximab-treated patients: $B$ cells promote humoral immune responses in the presence of T-cell-mediated immunity. Ann Rheum Dis 2021;80:1344-9

45 Werbel WA, Boyarsky BJ, Ou MT, et al. Safety and immunogenicity of a third dose of SARS-CoV-2 vaccine in solid organ transplant recipients: a case series. Ann Intern Med 2021. doi:10.7326/L21-0282. [Epub ahead of print: 15 Jun 2021]

46 Machado PM, Lawson-Tovey S, Hyrich K, et al. LB0002 COVID-19 vaccine safety in patients with rheumatic and musculoskeletal disease. Ann Rheum Dis 2021;80:199-200. 